\title{
Timeliness of electronic discharge summary sign-off for medical inpatients at Great Western Hospital, Swindon: a retrospective audit
}

\author{
Authors: William Thomas ${ }^{\mathrm{A}}$ and Azeem Ahmed $^{\mathrm{A}}$
}

\section{Introduction}

Timely communication between healthcare providers is crucial for providing safe and effective care, particularly in the era of increasingly complex medical and social needs. Electronic discharge summaries (EDS) conveying relevant information from inpatient admissions to patients' GPs are an integral part of such communication. NICE ${ }^{1}$ and Professional Record Standards Body $(P R S B)^{2}$ guidelines stipulate EDS standards, including that such documents be made available to GPs within 24 hours of discharge. The primary aim of our audit was to evaluate compliance of medical teams at the Great Western Hospital NHS Foundation Trust (GWH) with this quality standard.

\section{Methods}

A random sample of 109 patients who were discharged from the care of inpatient medical teams at GWH to community settings between 1 January 2019 and 11 August 2019 was provided by the audit department at GWH. We retrospectively reviewed the EDS of these patients on 6 September 2019, noting the date that the EDS sent electronically to GPs, compared with the date, time and day at the point of discharge. We divided patients into length of stay groups of short ( 48 hours or less), medium (3-7 days) or long (more than 7 days). The primary outcome was the percentage of patients with an EDS sent for GP review within 24 hours of discharge.

\section{Results}

109 patients were included for analysis. Of these, 107 had EDS available at the point of review $(98.2 \%)$. The quality standard was achieved for 68 patients $(64.2 \%)$, with a 1 day median delay from discharge to EDS, but a wide range of 0 to 82 days (excluding two cases where no EDS was available at the time of our review). This standard was achieved less frequently when patients were discharged over the weekend (15/35 cases, $42.9 \%$ ) compared with those discharged from Monday to Friday (53/74 cases, $71.6 \%)$. There were no significant differences in compliance with the standard according to time of discharge or length of stay. For

Authors: ${ }^{A}$ Great Western Hospitals NHS Foundation Trust, UK reasons which are difficult to fully explain, there was a marked deterioration in compliance with the standard during the month of April 2019 compared with previous and subsequent months.

\section{Discussion}

This sampled, retrospective audit suggests that medical patients discharged over the weekend in GWH are more likely to experience a delay in the EDS being made available to their GP than those discharged from Monday to Friday. Given the additional workload pressures on on-call medical teams at the weekend, this emphasises the need for inpatient teams to identify potentially dischargeable patients and complete as much as practical of their EDS before the weekend. GWH are currently conducting a wider quality improvement regarding EDS, which could include actions to emphasise this need.

\section{Conflicts of interest}

None declared.

\section{References}

1 National Institute for Health and Care Excellence. Transition between inpatient hospital settings and community or care home settings for adults with social care needs: NICE guideline [NG27]. NICE, 2015.

2 Professional Record Standards Body. Implementation guidance report: e-Discharge standard. PRSB, 2019. 